\title{
The Electrocardiogram Following Dynamic Cardiomyoplasty
}

\author{
Andrew Oehler, M.D. ${ }^{1}$ and Joseph Chiovaro, M.D. ${ }^{1,2}$ \\ 'Department of General Internal Medicine, Oregon Health \& Science University, Portland, OR, USA; ${ }^{2}$ Division of Hospital and Subspecialty Medicine, \\ Portland Veterans Affairs Medical Center, Portland, OR, USA.
}

\author{
KEY WORDS: heart failure; electrocardiography; cardiomyoplasty. \\ J Gen Intern Med 32(2):223-4 \\ DOI: $10.1007 / \mathrm{s} 11606-016-3827-7$ \\ (C) Society of General Internal Medicine 2016
}

\begin{abstract}
A 66-year-old man with ischemic cardiomyopathy status A post-dynamic cardiomyoplasty in 1994 and revision 2 months prior to admission presented with increasing erythema around the site of his muscle stimulator. An ultrasound of the stimulator pocket showed no fluid collection, and the patient was started on intravenous antibiotics. The ECG revealed artifactual spikes from the latissimus dorsi muscle (LDM) stimulated to contract in synchrony with cardiac systole: typical changes following dynamic cardiomyoplasty (Fig. 1).
\end{abstract}

Cardiomyoplasty is a surgical treatment for end-stage heart failure that is an alternative to heart transplant, whereby the LDM is pedunculated and wrapped around the heart (Fig. 2). This wrap is then "conditioned" by electrical stimulation to reduce fatigability, transforming the fast-twitch skeletal muscle into slow-twitch fibers similar to cardiac muscle. Dynamic cardiomyoplasty was hypothesized to improve systolic function via augmented contraction and to reduce remodeling by a girdling effect of the wrap, with most clinical benefit arising from the latter. The procedure fell out of favor in the USA, primarily due to (a) improvement in ejection fraction but neutral effect on invasive hemodynamics, ${ }^{2}$ (b) lack of longterm mortality improvement ${ }^{2}$ and (c) Medtronic withdrawal of their cardiomyostimulators in the late 1990s, leaving surgeons without an FDA-approved device. ${ }^{3}$

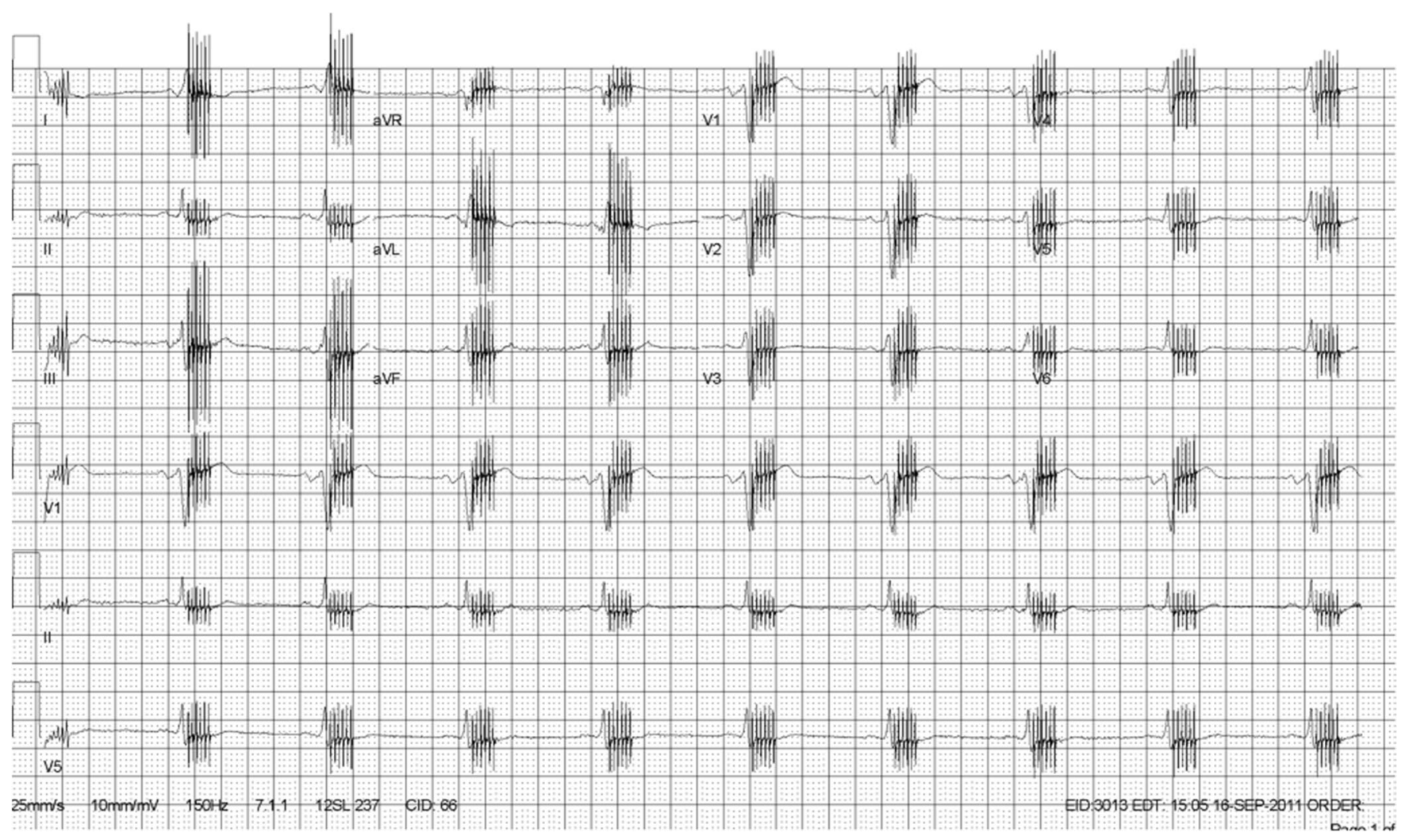

Figure 1 Electrocardiogram demonstrating artifact from latissimus dorsi stimulator.

Received July 1, 2016

Revised July 19, 2016

Accepted July 20, 2016

Published online August 3, 2016 


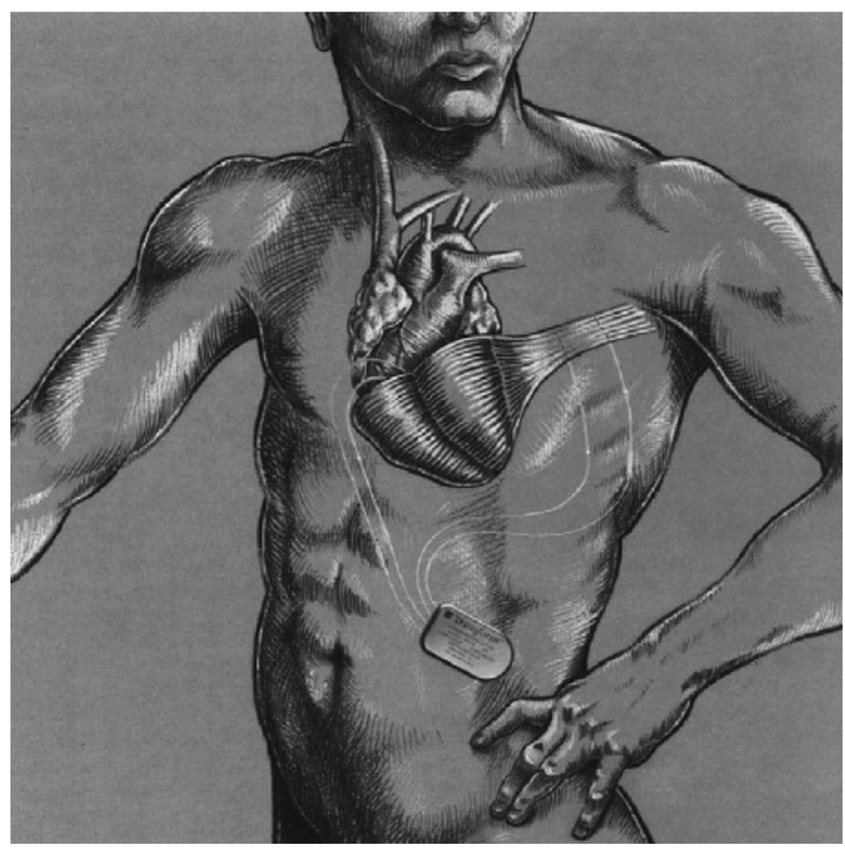

Figure 2 Dynamic cardiomyoplasty using the left latissimus dorsi muscle flap. (Image used with permission from J.C. Chachques et al. ${ }^{1}$ ).
Corresponding Author: Andrew Oehler, M.D.; Department of General Internal Medicine, Oregon Health \& Science University, Portland, OR, USA (e-mail: oehler@ohsu.edu).

\section{Compliance with Ethical Standards:}

Conflict of Interest: The authors declare that they do not have a conflict of interest.

\section{REFERENCES}

1. Chachques JC, et al. Heart transplantation following cardiomyoplasty: a biological bridge. Eur J Cardiothorac Surg. 2008;33:685-690.

2. Furnary AP, Jessup M, Moreira LFP. Multicenter trial of dynamic cardiomyoplasty for chronic heart failure. J Am Coll Cardiol. 1996;28:1175-1180.

3. Moreir LFP, Stolf NAG. Dynamic cardiomyoplasty as a therapeutic alternative: current status. Heart Fail Rev. 2001;6:201-212. 